\title{
Overexpression of high mobility group protein B1 correlates with the proliferation and metastasis of lung adenocarcinoma cells
}

\author{
KE-KANG SUN ${ }^{1}, \mathrm{CHENG} \mathrm{JI}^{2}, \mathrm{XING} \mathrm{LI}^{1}, \mathrm{LU}_{\mathrm{ZHANG}}{ }^{1}, \mathrm{JUN}^{\mathrm{DENG}}{ }^{1}$, NING ZHONG ${ }^{1}$ and XIAO-YANG WU ${ }^{1}$ \\ ${ }^{1}$ Department of Gastrointestinal Surgery, Thoracic Surgery Division, Kunshan First People's Hospital \\ Affiliated to Jiangsu University, Kunshan, Jiangsu 215300; ${ }^{2}$ Department of Respiratory Medicine, \\ First Affiliated Hospital to Soochow University, Suzhou, Jiangsu 215006, P.R. China
}

Received October 26, 2012; Accepted February 27, 2013

DOI: $10.3892 / \mathrm{mmr} .2013 .1362$

\begin{abstract}
High mobility group protein B1 (HMGB1) plays an important role in a number of clinical conditions, such as autoimmunity, cardiovascular disease and cancer. Evidence suggests that HMGB1 is critical in the development and progression of numerous types of tumor. However, the underlying molecular mechanisms for the HMGB1-mediated progression and metastasis of lung cancer have not yet been elucidated. In this study, we investigated the role of HMGB1 in lung adenocarcinoma and the mechanisms by which it contributes to carcinogenesis and metastasis. We demonstrated that there was an increase in the expression of HMGB1 in primary cancer tissues compared to the matched adjacent non-cancerous tissues. The expression levels of TOB1 in the normal human bronchial epithelial (HBE) cell line and 10 lung cancer cell lines were determined by reverse transcription-PCR (RT-PCR). The results revealed that HMGB1 expression increased in 8 cell lines compared with the HBE cell line. The A549 and NCI-H1975 cells were transfected with HMGB1 recombinant plasmid. We discovered that the overexpression of HMGB1 promoted cell growth and metastasis in the 2 cell lines. Further investigation revealed that exogenously expressed HMGB1 enhanced the activation of p38 and Erk1/2, in addition to the expression of nuclear factor $(\mathrm{NF})-\kappa \mathrm{B}$. We propose that HMGB1 functions as a tumor promoter and that it regulates the proliferation and invasion of lung cancer cells by modulating the activation of the Erk1/2 and p38 mitogen-activated protein kinase (MAPK) signaling pathways.
\end{abstract}

Correspondence to: Dr Ning Zhong, Department of Gastrointestinal Surgery, Thoracic Surgery Division, Kunshan First People's Hospital affiliated to Jiangsu University, 93 West Qianjin Road, Kunshan, Jiangsu 215300, P.R. China

E-mail: fsyx7skk@163.com

Key words: high mobility group protein B1, lung cancer, angiogenesis, metastasis, mitogen-activated protein kinase

\section{Introduction}

Lung adenocarcinoma accounts for approximately $40 \%$ of all lung cancer cases (1). A diverse range of genetic abnormalities are observed in lung cancer cells, but less common genetic changes are observed in lung adenocarcinoma $(2,3)$. Understanding the molecular processes underlying these cellular phenotypic changes is critical in order to develop novel therapeutic strategies. Key to mediating these changes are the interactions between cell surface receptors and their cognate ligands, which, through intracellular signaling, induce alterations in gene expression (4).

High mobility group protein B1 (HMGB1) is a highly conserved nuclear protein, acting as a chromatin-binding factor that binds DNA and promotes access to transcriptional protein assemblies on specific DNA targets (5-7). In addition to its nuclear role, HMGB1 also functions as an extracellular signaling molecule during inflammation, cell migration and tumor metastasis (8). The overexpression of HMGB1 has been associated with each of the hallmarks of cancer, including unlimited replicative potential, the ability to develop blood vessels, evasion of programmed cell death, tissue invasion and metastasis $(9,10)$.

In this study, by analyzing the expression of HMGB1 in lung adenocarcinoma tissues and lung cancer cell lines, we demonstrate that HMGB1 plays a role in the development of lung adenocarcinoma. Furthermore, we provide evidence suggesting that HMGB1 is involved in the regulation of angiogenesis, invasion and metastasis of lung adenocarcinoma cells.

\section{Materials and methods}

Lung cancer cell lines and patients. The normal human bronchial epithelial (HBE) cell line and 8 human lung cancer cell lines (95-D, A549, NCI-H1299, NCI-H1975, NCI-H661, NCI-H446, NCI-H1395 and Calu-3) were purchased from the American Type Culture Collection (Manassas, VA, USA). SPCA-1 and LTEP- $\alpha-2$ cell lines were obtained from Shanghai Institutes for Biological Sciences (Shanghai, China). Primary lung adenocarcinoma and corresponding non-cancerous tissues were obtained during surgery from 24 patients who were treated at Kunshan First People's Hospital affiliated 
to Jiangsu University (Kunshan, China). The materials to be analyzed were selected by a pathologist to ensure that macroscopically the samples were entirely cancerous and from an area devoid of necrotic tissue. Cancerous and non-cancerous tissues were stored at $-80^{\circ} \mathrm{C}$ until protein extraction. Patients provided written informed consent, and the study was approved by the ethics committee of Jiangsu University.

Cell culture and reagents. The human lung cancer cell lines, 95-D, A549, NCI-H1299, NCI-H1975, NCI-H661, NCI-H446, NCI-H1395, Calu-3, SPCA-1, LTEP- $\alpha-2$, and the HBE cell line were maintained in Dulbecco's modified Eagle's medium (DMEM) or RPMI-1640 supplemented with $10 \%$ fetal calf serum, L-glutamine ( $5 \mathrm{mmol} / \mathrm{l})$, non-essential amino acids $(5 \mathrm{mmol} / \mathrm{l})$, penicillin $(100 \mathrm{U} / \mathrm{ml})$ and streptomycin $(100 \mathrm{U} / \mathrm{ml})$ (Invitrogen, Carlsbad, CA, USA), at $37^{\circ} \mathrm{C}$ in a humidified $5 \% \mathrm{CO}_{2}$ atmosphere.

Plasmids and transfection. The HMGB1 eukaryotic expression vector construction and plasmid transfection using Lipofectamine were performed as previously described (11). The expression of HMGB1 was determined by western blot analysis.

Cell viability assay. Cell proliferation was performed by 3-(4,5-dimethylthiazol-2-yl)-2,5-diphenyltetrazolium bromide (MTT) viability assay, the most common assay for determining cell growth and death. The MTT survival assay has been described in detail in previous studies (12). Briefly, exponentially growing cells were recultured $(5,000$ cells/well) overnight in 96-well tissue culture plates. Up to $20 \mu \mathrm{l}$ of MTT (Sigma Aldrich, St. Louis, MO, USA) was added to the medium in each well, with a final concentration of $2 \mathrm{mg} / \mathrm{ml}$. After $4 \mathrm{~h}$ of incubation, the medium containing MTT was discarded, and $120 \mu \mathrm{l}$ of DMSO were added followed by incubation for $10 \mathrm{~min}$. The absorbance was measured using an ELISA reader at $570 \mathrm{~nm}$, with the absorbance at $630 \mathrm{~nm}$ taken as the background correction. Cell viability was expressed as a percentage of the untreated controls. All experiments were performed at least 3 times.

Transwell invasion assay. The invasion assay was performed using Transwell filters (Millipore, Billerica, MA, USA) as previously described (13). The Transwell filter surfaces (8 $\mu \mathrm{m}$ pore size) were uniformly coated with $25 \mathrm{mg}$ Matrigel overnight at $4^{\circ} \mathrm{C}$ prior to the experiment. The lower chamber was filled with culture medium containing $10 \%$ fetal calf serum, and the subconfluent proliferating cells were carefully transferred onto the coated upper surface of the chamber. After $24 \mathrm{~h}$ of incubation, the filter was gently removed, and the upper surface of the filter was wiped using a cotton swab to remove all attached cells. The cells that invaded the Matrigel and attached to the lower surface of the filter were fixed with $4 \%$ paraformaldehyde, stained with Giemsa, and then counted in 15 randomly selected microscopic fields per filter. Finally, the invasion rate was calculated and compared. Experiments were performed independently at least 3 times.

Western blot and immunoprecipitation/immunoblot analysis. Westernblotanalysis was performedaspreviously described(14).

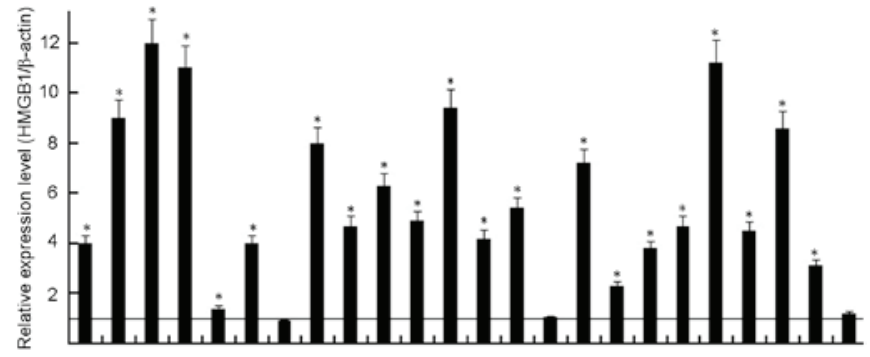

Figure 1. Increased HMGB1 expression in lung adenocarcinoma tissues. HMGB1 protein expression in 24 human primary lung adenocarcinomas was evaluated by western blot analysis with $\beta$-actin as the loading control. The data shown are the ratio of HMGB1 expression in each cancerous tissue to that of the corresponding non-cancerous tissue. ${ }^{*} \mathrm{P}<0.01$.

Thefollowing primary antibodieswere usedforimmunoblotting: $\beta$-actin (C-4), HMGB1 (E-1), nuclear factor (NF)- $\mathrm{B}$ (P65A) and secondary antibody horseradish peroxidase (HRP)-labeled goat anti-mouse (GAM-007), goat anti-rabbit (SC-2004) IgG, as well as phospho-ERK1/2 (T202/Y204), ERK1/2, phosphop38 (T180/Y182) and p38 (Cell Signaling Technology, Danvers, MA, USA, 1:1,000 dilution). The protein bands were quantified by densitometry using QuantityOne software (Bio-Rad, Hercules, CA, USA), and the values were expressed relative to $\beta$-actin (control for loading and transfer). At least 3 independent experiments were performed for each cell type examined.

Semi-quantitative reverse transcription-PCR (RT-PCR) analysis. mRNA expression was determined by RT-PCR. $\mathrm{PCR}$ reaction conditions and cycle numbers were rigorously adjusted so that each reaction occurred within the linear range of amplification. Detailed methods for RNA isolation, cDNA synthesis and RT-PCR analyses have been previously described (15). The primers for gene-specific PCR were as follows: GAPDH sense, 5'-CAA CTA CAT GGT CTA CAT GTT CC-3' and antisense, 5'-CAA CCT GGT CCT CAG TGT AG-3'; and HMGB1 sense, 5'-TTA GAT AGC CCT GTC CTG GTG G-3' and antisense, 5'-TGA ATG TGG CAT CTT TGT TTG A-3'. PCR products were analyzed by electrophoresis using $1 \%$ agarose gels containing $0.1 \mathrm{mg} / \mathrm{ml}$ ethidium bromide and the gels were photographed under ultraviolet light. mRNA expression levels were quantified by densitometry of the cDNA bands using QuantityOne software (Bio-Rad). At least 3 independent experiments were performed for each cell type examined.

Statistical analysis. The data are presented as the means \pm SD. Statistical comparisons of the experimental results between the treated group and the control group were made using the two-tailed Student's t-test. All statistical tests were performed using SPSS version 17.0 software. $\mathrm{P} \leq 0.01$ was considered to indicate a statistically significant difference.

\section{Results}

Increased expression of HMGB1 in lung adenocarcinoma specimens. In order to elucidate the role of HMGB1 in lung cancer, we examined the level of HMGB1 expression in lung adenocarcinoma cells by western blot analysis using 


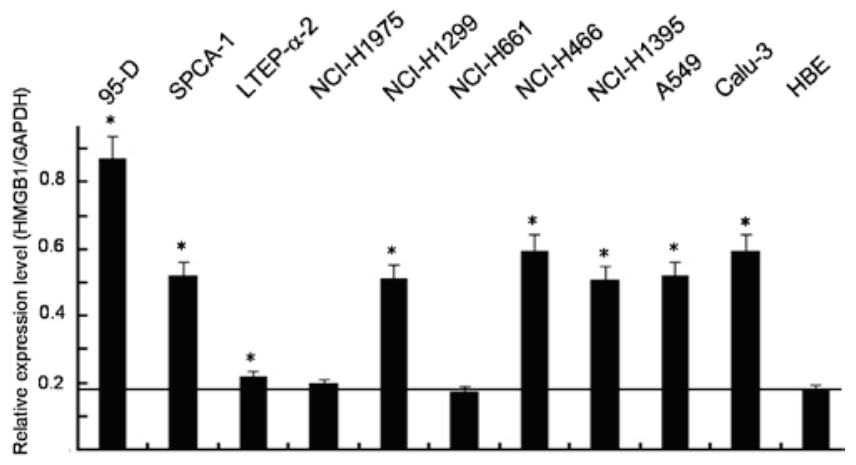

Figure 2. HMGB1 protein expression in various cell lines was examined by RT-PCR. The mRNA expression of HMGB1 in the normal human bronchial epithelial (HBE) cell line and 10 lung cancer cell lines was evaluated by RT-PCR.GAPDH was used as the loading control. The data shown are the ratio of HMGB1 expression in each cancer cell line to that of HBE cells. "P<0.01.
$\mathbf{A}$

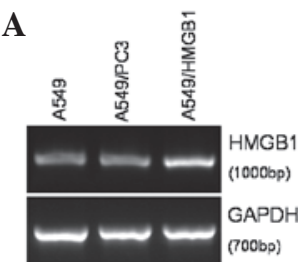

B

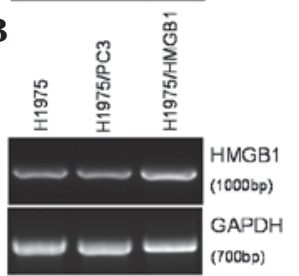

C

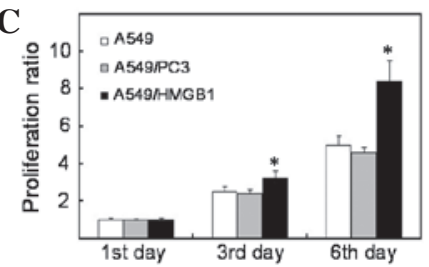

D

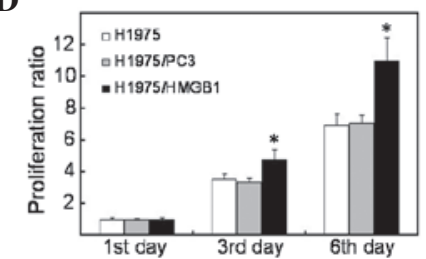

E
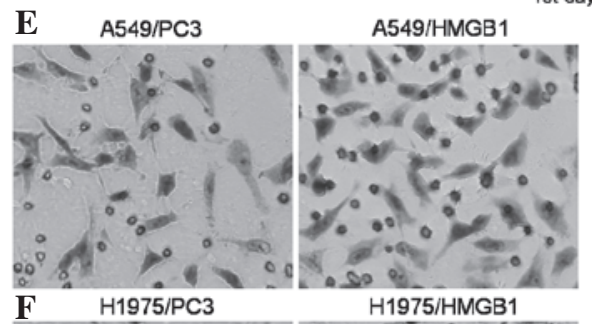
H1975/HMGB1
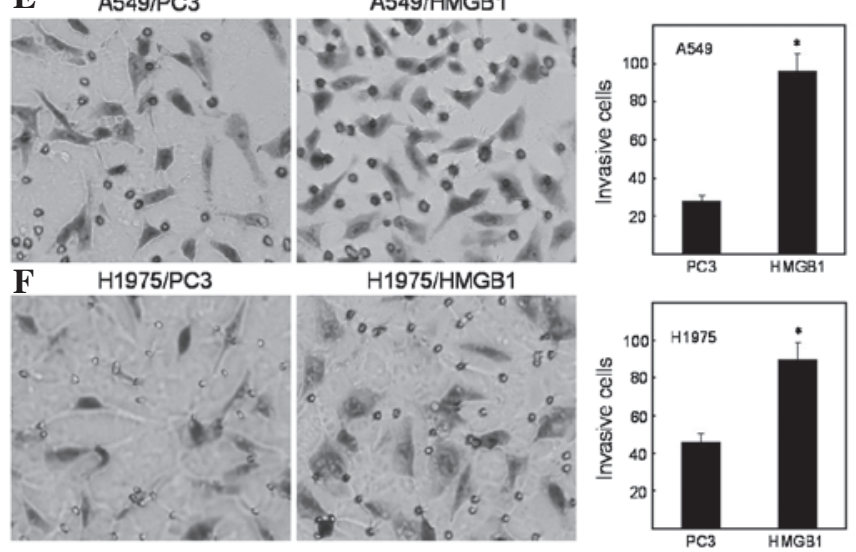

Figure 3. Exogenous expression of HMGB1 promotes cell proliferation and invasion. (A and B) The overexpression of HMGB1 in the transfected lung cancer cells was confirmed by RT-PCR. GAPDH was used as the loading control. (C and D) Exogenous expression of HMGB1 promoted lung cancer cell proliferation, as shown by MTT viability assays. Cells were counted on days 1 , 3 and 6. The proliferation rate was measured as fold changes in cell growth. (E and F) The cell invasion induced by HMGB1 was analyzed by Transwell assay. After $24 \mathrm{~h}$ of incubation, the invaded cells attached to the lower surface were fixed, stained and photographed under a phase contrast microscope and then counted in 10 randomly selected microscopic fields. $\mathrm{P}<0.01$.

clinical samples. A total of 24 lung adenocarcinoma cases were examined. In each patient, the level of HMGB1 expression normalized to $\beta$-actin expression in cancerous tissue was compared to that observed in non-cancerous tissue. In
A

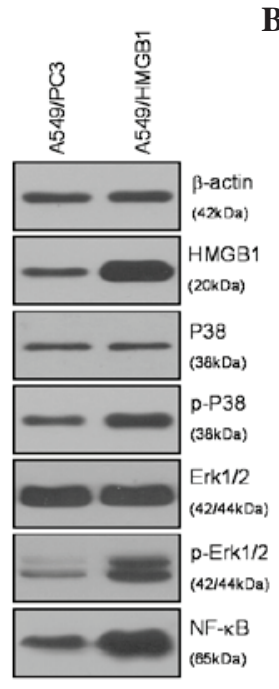

B

C

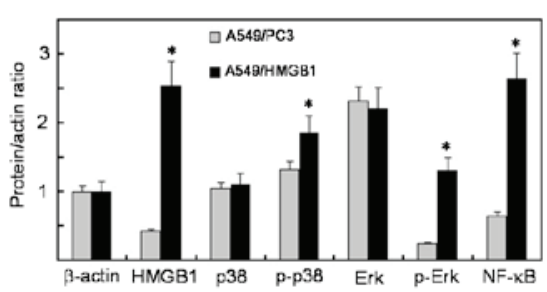

D

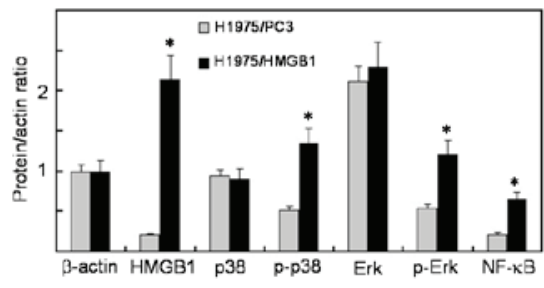

Figure 4. Overexpression of HMGB1 activates the ERK and p38 MAPK signaling pathway. (A and B) Western blot analysis was performed to determine the expression levels of Erk1/2, p38, phosphorylated Erk1/2 (p-Erk1/2), phosphorylated p38 (p-p38) and the downstream NF- $\kappa$ B cascade in the HMGB1-overexpressing A549 cells, as well as in the NCI-H1975 cells. $\beta$-actin was used as the loading control. (C and D) The expression levels are displayed as fold changes in band density. Asterisk indicates a statistical difference between experimental groups $\left({ }^{*} \mathrm{P}<0.01\right)$.

$87.5 \%(21 / 24)$ of the specimens, HMGB1 expression increased in the cancerous tissues compared to the non-cancerous tissues. The protein bands were quantified using QuantityOne software, and the augmentation was deemed to be statistically significant $(\mathrm{P}<0.01)$ (Fig. 1). Therefore, we hypothesized that the overexpression of HMGB1 contributes to the development of human lung adenocarcinoma. However, due to the fact that we analyzed only a small number of specimens, future analysis with a much larger number of specimens is required.

HMGB1 overexpression in lung cancer cell lines. To determine the effect of HMGB1 on the proliferation and metastasis of lung cancer cells, the HMGB1 expression levels in the 10 lung cancer cell lines and the HBE cell line were analyzed by RT-PCR. As shown in Fig. 2, the HMGB1 protein level increased in 8 of the lung cancer cell lines compared to the HBE cell line and there was little or no change in the expression levels of GAPDH in these cell lines. In vitro data confirmed that TOB1 overexpression is an important event in lung cancer. 
Exogenous expression of HMGB1 enhances the proliferation and invasion of lung cancer cells. Cell proliferation and invasion are indispensable for cancer metastasis. Due to the fact that HMGB1 expression was increased in the lung cancer tissues and cell lines, we hypothesized that HMGB1 regulates the progression of lung cancer. According to gain-of-function approaches, A549 and NCI-H1975 cells were selected as model systems, as they are lung adenocarcinoma cell lines that express almost undetectable levels of HMGB1. Moreover, A549 cells are epidermal growth factor receptor (EGFR) wild-type and NCI-1975 cells are EGFR mutated, and EGFR status is known to modulate proliferation and metastasis. Using Lipofectamine and G418-mediated plasmid stable transfection, multiple clones transfected with HMGB1 were selected and confirmed by RT-PCR (Fig. 3A and B). The dynamics of A549, A549/PC3 and A549/HMGB1 cell growth were determined by MTT assay. Following a 6-day period, the overexpression of HMGB1 enhanced the proliferative ability of the A549 cells, compared to the control groups. The marked decrease in cell viability was observed on day 3 (Fig. 3C). We examined the effect of HMGB1 expression on the invasive ability of A549 cells using a Transwell assay. The results demonstrated that, compared to the parental cells, the overexpression of HMGB1 enhances the ability of the A549 cells to invade through the Matrigel-coated filter. As shown in Fig. 3E, the invasion rate of A549/HMGB1 cells increased by $>60 \%$ corresponding to the vector-transfected cells $(\mathrm{P}<0.01)$. In the EGFR-mutated NCI-H1975 cells, the overexpression of HMGB1 also promoted cell proliferation and invasion (Fig. 3D and F).

HMGB1 activates the ERK and 38 mitogen-activated protein kinase (MAPK) signaling pathway. To determine whether plasmid transfection induces the activation of Erk $1 / 2$ and p38 MAPK, we transfected the A549 cells with HMGB1 recombinant plasmid and then immunoblot analysis was performed to determine the phosphorylation levels of Erk1/2 and p38. The results demonstrated that HMGB1 overexpression enhanced the expression levels of phosphorylated Erk1/2 and p38; however, the levels of total Erk1/2 and p38 remained unaffected. These data suggest that Erk1/2 and p38 MAPK are activated in HMGB1-transfected lung cancer cells. The MAPKs integrate a wide range of upstream signals to determine patterns of downstream gene expression through the regulation of transcription factors. NF- $\mathrm{KB}$ has been well characterized as a molecule regulated by the MAPK signaling pathway (16). In this study, the expression of NF- $\mathrm{BB}$ was upregulated in HMGB1-overexpressed A549 cells (Fig. 4A and C). As for EGFR-mutated NCI-H1975 cells, the overexpression of HMGB1 activated the ERK and p38 MAPK signaling pathways when compared with the control cells, implying that HMGB1 activated the ERK and p38 MAPK signlaing pathways through an EGFR-independent pathway (Fig. 4B and D).

\section{Discussion}

Although lung cancer has the highest mortality rate of all cancers worldwide, a number of developments indicate future clinical benefits $(17,18)$. Perhaps the most significant advance in the treatment of this disease has been the introduction of molecularly targeted therapies, a term that currently includes monoclonal antibodies and small-molecule tyrosine kinase inhibitors. The development of effective targeted therapeutics requires knowledge of the genes and pathways involved and the mechanisms by which they affect the biological behavior of lung cancer (4).

In this study, we identified the increased expression of HMGB1 protein in lung adenocarcinoma tissues and lung cancer cell lines, which suggests that the expression of HMGB1 is biologically significant in lung cancer development. We further demonstrated that the enforced overexpression of HMGB1 modulated the proliferation and invasion of 2 lung adenocarcinoma cell lines, A549 and NCI-H1975, in vitro. These observations support our hypothesis that HMGB1 plays an oncogenic role in lung cancer development. Additionally, using western blot analysis, we identified the activation of the Erk $1 / 2$ and p38 cascade, as evidenced by the phosphorylation of ERK1/2 and p38, revealing the role of HMGB1 in the regulation of the ERK1/2 and p38 MAPK pathway. Consistently, ERK1/2 and $\mathrm{p} 38$ activation leads to the transcriptional regulation of $\mathrm{NF}-\mathrm{kB}$, resulting in increased cell proliferation and invasion, suggesting that MAPK/NF- $\mathrm{KB}$ is an oncogenic mechanism by which HMGB1 contributes to lung cancer development.

In conclusion, lung adenocarcinoma cell growth and survival is impaired by the inactivation of HMGB1, suggesting that HMGB1 plays an oncogenic role and is a potentially novel therapeutic target.

\section{Acknowledgements}

This study was supported by grants from the Natural Science Foundation of Jiangsu Province (SZ126821), the Social Development Projects of Kunshan City (KS1224) and the Priority Academic Program Development of Jiangsu Higher Education Institutions (PAPD).

\section{References}

1. Jemal A, Bray F, Center MM, et al: Global cancer statistics. CA Cancer J Clin 2: 69-90, 2011.

2. Singhal S, Miller D, Ramalingam S and Sun SY: Gene expression profiling of non-small cell lung cancer. Lung Cancer 60: 313-324, 2008.

3. Li C, Fan S, Owonikoko TK, et al: Oncogenic role of EAPII in lung cancer development and its activation of the MAPK-ERK pathway. Oncogene 35: 3802-3812, 2011.

4. Larsen JE, Cascone T, Gerber DE, et al: Targeted therapies for lung cancer. Cancer J 6: 512-527, 2011.

5. Lotze MT and Tracey KJ: High-mobility group box 1 protein (HMGB1): nuclear weapon in the immune arsenal. Nat Rev Immunol 5: 331-342, 2005.

6. Muller S, Scaffidi P, Degryse B, et al: New EMBO members' review: the double life of HMGB1 chromatin protein: architectural factor and extracellular signal. EMBO J 20: 4337-4340, 2001.

7. Tang DL, Kang R, Zeh HR III and Lotze MT: High-mobility group box 1 and cancer. Biochim Biophys Acta 1799: 131-140, 2010.

8. Lotze MT and DeMarco RA: Dealing with death: HMGB1 as a novel target for cancer therapy. Curr Opin Investig Drugs 4: $1405-1409,2003$.

9. Ito I, Fukazawa J and Yoshida M: Post-translational methylation of high mobility group box 1 (HMGB1) causes its cytoplasmic localization in neutrophils. J Biol Chem 282: 16336-16344, 2007.

10. Taguchi A, Blood DC, del Toro G, et al: Blockade of RAGE-amphoterin signalling suppresses tumour growth and metastases. Nature 405: 354-360, 2000. 
11. Jiao Y, Wang HC and Fan SJ: Growth suppression and radiosensitivity increase by HMGB1 in breast cancer. Acta Pharmacol Sin 28: 1957-1967, 2007.

12. Li XL, Meng QH and Fan SJ: Adenovirus-mediated expression of UHRF1 reduces the radiosensitivity of cervical cancer HeLa cells to gamma-irradiation. Acta Pharmacol Sin 30: 458-466, 2009.

13. Wu QF, Liu C, Tai MH, et al: Knockdown of FoxM1 by siRNA interference decreases cell proliferation, induces cell cycle arrest and inhibits cell invasion in MHCC-97H cells in vitro. Acta Pharmacol Sin 31: 361-366, 2010.

14. Jiao Y, Ge CM, Meng QH, et al: Adenovirus-mediated expression of Tobl sensitizes breast cancer cells to ionizing radiation. Acta Pharmacol Sin 28: 1628-1636, 2007.
15. Jiao Y, Ge CM, Meng QH, et al: Dihydroartemisinin is an inhibitor of ovarian cancer cell growth. Acta Pharmacol Sin 28: 1045-1056, 2007.

16. Jiang R, Wang NP, Tanaka KA, et al: Factor Xa induces tissue factor expression in endothelial cells by P44/42 MAPK and NF-кB-dependent pathways. J Surg Res 169: 319-327, 2011.

17. Ray MR, David J and He B: Lung cancer therapeutics that target signaling pathways: an update. Expert Rev Respir Med 4: 631-645, 2010.

18. Palmer RH, Vernersson E, Grabbe C and Hallberg B: Anaplastic lymphoma kinase: signalling in development and disease. Biochem J 420: 345-361, 2009. 